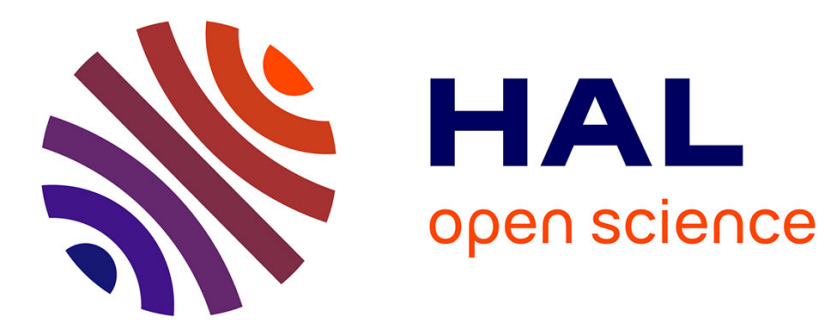

\title{
Internal waves generated by a translating and oscillating sphere
}

Pascal Dupont, Bruno Voisin

\section{To cite this version:}

Pascal Dupont, Bruno Voisin. Internal waves generated by a translating and oscillating sphere. Dynamics of Atmospheres and Oceans, 1996, 23 (1-4), pp.289-298. 10.1016/0377-0265(95)00436-X . hal-01943526

\section{HAL Id: hal-01943526 \\ https://hal.science/hal-01943526}

Submitted on 3 Dec 2018

HAL is a multi-disciplinary open access archive for the deposit and dissemination of scientific research documents, whether they are published or not. The documents may come from teaching and research institutions in France or abroad, or from public or private research centers.
L'archive ouverte pluridisciplinaire HAL, est destinée au dépôt et à la diffusion de documents scientifiques de niveau recherche, publiés ou non, émanant des établissements d'enseignement et de recherche français ou étrangers, des laboratoires publics ou privés. 


\title{
Internal waves generated by a translating and oscillating sphere
}

\author{
Pascal Dupont ${ }^{1}$, Bruno Voisin * \\ LEGI/IMG, CNRS-UJF-INPG, BP 53, 38041 Grenoble Cedex 9, France
}

(Received ??; revised ??; accepted ??)

\section{ABSTRACT}

At high Reynolds and Froude numbers, lee waves due to the horizontal motion of a body in a stratified fluid are superseded by random waves generated by its wake. The origin of these waves lies in the buoyant collapse of the large-scale coherent structures of the wake, and can be modelled as a source moving at the velocity of the body and of strength oscillating at the frequency of vortex shedding. In the present paper two parallel studies of the associated wave field are described. The first of these is theoretical and considers localized and extended models of the source, while the second is experimental and involves a vertically oscillating and horizontally translating sphere. Oscillation frequencies both smaller and larger than the Brunt-Väisälä frequency are considered, and reasonably good agreement between theory and experiment is obtained concerning, e.g., the shape of the surfaces of constant phase, the streamwise evolution of the wavelength, and the domain of existence of the waves. Calculations are then presented for a realistic turbulent wake, and comparison with available experimental results is performed.

* Corresponding author

1 Also at SPEA/GMEI/CNRM, Météo-France, 42 av. Coriolis, 31057 Toulouse Cedex, France 


\section{INTRODUCTION}

A body moving horizontally in a stratified fluid generates several systems of internal waves, each of which is associated with a distinct perturbation of the basic stratification. In this respect, the important parameters are the Froude number $\mathrm{Fr}=U / \mathrm{Na}$, which represents the ratio of inertial forces to buoyancy forces, and the Reynolds number $R e=U l / v$, which represents the ratio of inertial forces to viscous forces, with $U$ the velocity of the body, $a$ its transverse radius, $l$ its axial length, $N$ the Brunt-Väisälä frequency and $v$ the kinematic viscosity. In particular, experiments over the past twenty years (Lin and Pao, 1979; Gilreath and Brandt, 1985; Hopfinger et al., 1991; Bonneton et al., 1993; Lin et al., 1993) have shown that, at sufficiently high $F r$ and $R e$, lee waves generated by the motion of the body are superseded by random waves generated by its wake, and have traced back the origin of these waves to the large-scale coherent structures of the wake.

The modelling of this phenomenon was discussed by Voisin (1994b). In the Reynolds and Froude numbers range involved, the wake is turbulent and develops initially as in a homogeneous fluid. Coherent structures, in the form of vortex loops or turbulent bursts, are released periodically behind the body at the frequency $\omega_{0}$ of the near wake spiral instability; after a dimensionless time $N t_{\mathrm{c}} \approx 3$ they collapse impulsively under the influence of buoyancy, generating internal waves. As far as these waves are concerned, a turbulent wake is thus amenable to a series of impulses, of alternating signs so as to take into account its geometry, periodically spaced in both space and time, and separated from one another by a distance $\pi U / \omega_{0}$ and time $\pi / \omega_{0}$. Two equivalent views of this series arise naturally: a source moving at velocity $U$ and emitting impulses at time intervals $\pi / \omega_{0}$; a source moving at velocity $U$ and of strength oscillating at the frequency 
$\omega_{0}$ and at all its odd harmonics. The first view is appropriate at small axial distances from the body, when the waves generated by each collapse have not yet had time to interfere; then each impulse can be considered individually. Conversely, the second view is appropriate at large axial distances, when interference has taken place; then the collective effect of all the impulses is observed, resulting in a predominance of the fundamental frequency $\omega_{0}$.

A first step towards assessment of this interpretation was carried out by Bonneton et al. (1993), who studied each coherent structure individually. The present paper investigates the second, collective, aspect. Specifically, Section 2 describes a theoretical approach of internal waves generated by the simplest practical realization of a horizontally translating source of oscillatory strength, i.e., a sphere both translating horizontally and oscillating vertically. Then Section 3 compares the results of this theory with experiments, and Section 4 applies them to a realistic turbulent wake.

\section{THEORETICAL BACKGROUND}

\subsection{Source model}

In a homogeneous fluid, a sphere of radius $a$ moving at velocity $\mathbf{v}_{0}(t)$ along a path $\mathbf{r}_{0}(t)$, with $\mathbf{v}_{0}(t)=\mathrm{d} \mathbf{r}_{0} / \mathrm{d} t$, can be modelled either approximately, for $R \gg a$, as the dipole $m_{\mathrm{d}}(\mathbf{r}, t)=-2 \pi a^{3} \mathbf{v}_{0} \cdot \nabla \delta(\mathbf{R})$,

or exactly, for all $R / a$, as the surface distribution of monopoles

$m_{\mathrm{s}}(\mathbf{r}, t)=\frac{3}{2} \mathbf{v}_{0} \cdot \frac{\mathbf{R}}{a} \delta(R-a)$,

where $\mathbf{r}=[x, y, z]$ denotes position, with $r=|\mathbf{r}|, t$ denotes time, $\mathbf{R}=\mathbf{r}-\mathbf{r}_{0}$ is the position

relative to the centre of the sphere, $\mathbf{v}=\left[v_{x}, v_{y}, v_{z}\right]$ the fluid velocity and $m=\nabla \cdot \mathbf{v}$ the 
source strength (rate of volume outflow from the source) per unit volume. Use of these models in a stratified fluid was introduced by Miles (1971) and Gorodtsov and Teodorovich (1982), respectively, on the assumption that the Froude number be large, so that the flow around the sphere is locally unaffected by gravity. Experiments suggest, however, that these models remain valid even for moderate values of $F r$, the only difference being the replacement of the far-field condition $R \gg a$ by the compactness condition $a \ll \lambda$, with $\lambda$ the wavelength of the waves (see, e.g., Voisin 1994a). Another approximation implicit in (1) and (2) is that the Reynolds number $R e$ be moderate, so that no wake is formed.

A motion composed of horizontal translation at velocity $U$ and vertical oscillation of frequency $\omega_{0}$ and amplitude $h$ corresponds to the path $\mathbf{r}_{0}(t)=-U t \mathbf{e}_{x}-h \sin \left(\omega_{0} t\right) \mathbf{e}_{z}$, where the $x$ and $z$-axes are horizontal and vertical, respectively, and of unit vectors $\mathbf{e}_{x}$ and $\mathbf{e}_{z}$. The change $x_{1}=x+U t$ defines a system of coordinates, shown in Fig. 1(a), of origin the mean position $O_{1}$ of the centre of the sphere and $x_{1}$-axis opposite to the mean motion. Small oscillations $h \ll a$ can be neglected in the position of the source but not in its velocity, so that (1) and (2) become

$$
\begin{aligned}
& m_{\mathrm{d}}(\mathbf{r}, t)=2 \pi a^{3}\left[U \frac{\partial}{\partial x}+h \omega_{0} \cos \left(\omega_{0} t\right) \frac{\partial}{\partial z}\right] \delta\left(x_{1}\right) \delta(y) \delta(z), \\
& m_{\mathrm{s}}(\mathbf{r}, t)=-\frac{3}{2}\left[U \frac{x_{1}}{a}+h \omega_{0} \cos \left(\omega_{0} t\right) \frac{z}{a}\right] \delta\left(r_{1}-a\right) .
\end{aligned}
$$

This approximation is straightforward for the surface source; for the dipole it follows from remarking that $m_{\mathrm{s}}(\mathbf{r}, t)$ is asymptotic to $m_{\mathrm{d}}(\mathbf{r}, t)$ in the limit of large wavelengths $\lambda \gg a$, as can be seen by comparing the spectra

$$
\begin{aligned}
& m_{\mathrm{d}}(\mathbf{k}, t)=-2 \mathrm{i} \pi a^{3}\left[U k_{x}+h \omega_{0} \cos \left(\omega_{0} t\right) k_{z}\right] \\
& m_{\mathrm{s}}(\mathbf{k}, t)=-6 \mathrm{i} \pi a^{3}\left[U k_{x}+h \omega_{0} \cos \left(\omega_{0} t\right) k_{z}\right] \frac{j_{1}(k a)}{k a},
\end{aligned}
$$


defined by

$m(\mathbf{r}, t)=\frac{1}{(2 \pi)^{3}} \int m(\mathbf{k}, t) \mathrm{e}^{-\mathrm{i} \mathbf{k} \cdot \mathbf{r}} \mathrm{d}^{3} k$,

where $\mathbf{k}=\left[k_{x}, k_{y}, k_{z}\right]$ is the wavenumber vector, with $k=|\mathbf{k}|=2 \pi / \lambda$, and $j_{1}(z)=(\sin z) / z^{2}-$ $(\cos z) / z$ is the spherical Bessel function of order 1 .

The sphere can thus be modelled as the linear superposition of two sources, both in uniform horizontal motion, and whose strengths are constant and oscillatory, respectively. For oscillation velocities $h \omega_{0}$ large compared with the translation velocity $U$ the second component becomes large compared with the first component (i.e., lee waves); in what follows this is only the situation that we shall consider and lee waves will accordingly be omitted. In terms of the dimensionless parameters introduced, e.g., by Davies et al. (1994), we have: moderate Reynolds number $\operatorname{Re}=2 U a / v$, large Froude number $F r=U / N a$, small Keulegan-Carpenter number $K e=h / a$ and large velocity ratio $h \omega_{0} / U$.

\subsection{Wave field}

Internal waves generated by the uniform horizontal motion of a source of oscillatory strength have been studied both theoretically (Stevenson and Thomas, 1969; Redekopp, 1975; Rehm and Radt, 1975; Peat and Stevenson, 1975) and experimentally (Stevenson and Thomas, 1969; Peat and Stevenson, 1975; Davies et al., 1994). Emphasis was on the determination of the surfaces of constant phase. Here we adopt the approach exposed in Voisin (1994a), in which the amplitude and the phase are obtained jointly.

Waves are ruled by the frequency ratio $\Upsilon=\omega_{0} / N$, and are expressed in terms of the auxiliary 
variable $\xi$, which in spherical coordinates $\left(r_{1}, \theta_{1}, \varphi_{1}\right)$ (see Fig. 1(a)) satisfies the equation

$\tan \theta_{1}=\frac{\xi^{2}\left(\sin ^{2} \varphi_{1}-\xi^{2}\right)^{\frac{1}{2}}}{\Upsilon \sin ^{2} \varphi_{1}-\xi^{3}} \operatorname{sign}(\Upsilon-\xi)$,

subject to the condition $|\xi|<\left|\sin \varphi_{1}\right|$. The solution to this equation defines two systems of waves, sum waves with positive $\xi$ and difference waves with negative $\xi$, each of which system is itself separated into transverse and divergent components. Both systems are contained within fronts, parts of which are caustics, and which correspond to the maxima or jumps ( $\xi=\Xi_{ \pm}, \theta_{1}=\Theta_{ \pm}$) observed in Fig. 1(b). The analytical expression of $\Xi_{ \pm}$and $\Theta_{ \pm}$was given in Voisin (1994a) and will not be repeated here. For each wave system the divergent and transverse components merge on the caustic; associated ranges of $\xi$ are $-\left|\sin \varphi_{1}\right|<\xi<\Xi_{-}$for divergent difference waves, $\Xi_{-}<\xi<0$ for transverse difference waves, $0<\xi<\Xi_{+}$for transverse sum waves and $\Xi_{+}<\xi<\left|\sin \varphi_{1}\right|$ for divergent sum waves.

The name of those components refers to the shape of their horizontal curves of constant phase, shown in Fig. 2(a). For $\Upsilon>1$ difference waves are upstream facing and sum waves downstream facing; both of them have cusps on the caustic where divergent and transverse waves meet, and extend only downstream. For $\Upsilon<1$ difference waves and transverse sum waves remain essentially unchanged, while divergent sum waves, starting up from the caustic, tend towards infinity downstream as $|y| \rightarrow|z|\left(1-\Upsilon^{2}\right)^{\frac{1}{2}} / \Upsilon$, come then back towards regions of smaller $x_{1}$ and finally close up upstream.

The derivation of the characteristics of the waves is straightforward and yields, for the frequency and wavenumber vector,

$\omega=N \xi, \quad \mathbf{k}=\frac{N}{U}\left[\Upsilon-\xi, \xi \frac{|\Upsilon-\xi| \cos \varphi_{1}}{\left(\sin ^{2} \varphi_{1}-\xi^{2}\right)^{\frac{1}{2}}},-\frac{1-\xi^{2}}{\xi} \frac{|\Upsilon-\xi| \sin \varphi_{1}}{\left(\sin ^{2} \varphi_{1}-\xi^{2}\right)^{\frac{1}{2}}}\right]$ 
The vertical displacement $\zeta$, related to the vertical velocity $v_{z}$ by $v_{z}=\partial \zeta / \partial t$, follows similarly from replacing in Voisin (1994a) the monopole strength $m_{0}$ of the source by the spectrum $m(\mathbf{k}, t)$. The resulting expressions, combining (5), (6) and (9), fail to describe the vicinity of the caustics, where they diverge; a more elaborate description of this vicinity, involving Airy functions (Lighthill, 1978, Section 4.11), was not attempted.

\section{SPHERE EXPERIMENTS}

Experiments were conducted in a transparent tank $50 \mathrm{~cm}$ wide, $50 \mathrm{~cm}$ deep and $400 \mathrm{~cm}$ long. A heavy sphere of radius $1.12 \mathrm{~cm}$ was towed horizontally through a linearly stratified fluid, a vertical sinusoidal oscillation being superimposed on the uniform horizontal translation of the support of the sphere. The towing and visualization techniques were identical to those used by Bonneton et al. (1993). Five frequency ratios $\Upsilon=0.4,0.6,0.8,1.01$ and 1.2, both smaller and larger than 1, were selected. Other parameters were chosen so that three of the four conditions mentioned in Section 2.1 be satisfied: moderate Reynolds number, small Keulegan-Carpenter number, large velocity ratio. The Froude number was then fixed by experimental requirements.

Experimental results are summarized in Fig. 2(d). Two factors appear to distort them significantly: the presence of lee waves, easily recognized by their hyperbolic curves of constant phase, and the reflection of the waves on the walls of the tank. Lee waves are all the more pronounced as $\Upsilon$ is small, since the velocity ratio $h \omega_{0} / U=\Upsilon K e / F r$ is, for given $F r$ and $K e$, proportional to $\Upsilon$. For a discussion of the effects and occurrence of tank wall reflections the reader is referred to Graham and Graham (1980).

The first conclusion to be drawn from Fig. 2 is the inadequacy of the dipole representation 
(3) of the sphere, since the displacement field shown in Fig. 2(b) is dominated by high-amplitude difference waves which are absent from experiments. The reason for this lies in the compactness condition $a \ll \lambda$, which is not satisfied by the short difference waves. Alternatively, this can be explained by remarking that the dipolar spectrum (5) varies as $k a$, overemphasizing the contribution of large wavenumbers, while the surface source spectrum (6) varies as $j_{1}(k a)$ and is thus a maximum at $k a \approx 2$ (Abramowitz and Stegun, 1972, Chapter 10). Only the surface source model can thus be expected to yield satisfactory agreement with experiments.

This agreement is first qualitative, and concerns the structure of the wave field. As shown in Fig. 2(d), divergent sum waves are dominant for $\Upsilon=0.4$; as $\Upsilon$ increases and reaches 0.8 transverse sum waves appear at some distance downstream, and finally for $\Upsilon=1.2$ they supersede divergent waves in the vicinity of the sphere. This results from the combination of several factors: position of the wavefronts $\theta_{1}=\Theta_{ \pm}$within which each wave system is found; amplitude associated with these systems in the spectrum $m(\mathbf{k}, t)$ of the source; restrictions imposed on the domain of observation of the waves by the presence of lee waves and by tank wall reflections. These arguments can be made quantitative, e.g., by plotting the function $j_{1}(k a)$ as a function of $x_{1}$ for $y=0$ (not shown here). As $\Upsilon$ increases from 0.4 to 1.2 , zones of rapid phase variations (nay inversions) appear near the caustics. This is attributable to an interference between transverse and divergent waves, of similar wavelengths in that zone.

The evolution of the wavefronts with $\Upsilon$, i.e., their narrowing as $\Upsilon$ increases, is confirmed by experiment. Their position is difficult to determine because of the perturbation of the wave field by the lee waves and by the wall reflections. For relatively small $x_{1}$, however, this perturbation is negligible, and the theoretical and experimental wavefronts differ by a lateral distance of the same order as the diameter of the sphere. A possible explanation for this is the diffraction of 
the waves outside the caustics, a phenomenon not taken into account in the formulae used to compute the theoretical wave field.

More quantitatively, Fig. 3(a) compares, for $\Upsilon=0.6$, the theoretical and experimental values of the axial wavelength $\lambda_{x}=2 \pi /\left|k_{x}\right|$ in the plane $y=0$, measured from several images similar to Fig. 2(d). The number of experimental runs compensates for the relatively poor precision of the method. Good agreement is obtained with the interpretation of the wave field in terms of mainly divergent sum waves. As $N x_{1} / U \rightarrow \infty$ we have for divergent difference waves, transverse waves and divergent sum waves, respectively,

$\lambda_{x} \sim 2 \pi \frac{U}{\omega_{0}}\left(\frac{1}{\Upsilon+1}, 1, \frac{1}{|\Upsilon-1|}\right)$

indicating that the axial wavelength of transverse waves tends towards the spatial period $2 \pi U / \omega_{0}$ of the path of the source. Figure 3(a) shows that this period is never observed in the wave field.

\section{WAKE APPLICATION}

Application of the preceding analysis to a realistic stratified turbulent wake, created by horizontal motion at velocity $U^{\prime}$ of a body of transverse radius $a^{\prime}$ and axial length $l^{\prime}$, supposes that the various parameters arising in this analysis be expressed in terms of the parameters associated with the wake. According to the discussion of Section 1, the velocity $U$ and frequency $\omega_{0}$ of the source equivalent to the wake are identical to the velocity $U^{\prime}$ of the body and to the frequency $\omega_{0}^{\prime}$ of the near wake spiral instability. Similarly, the radius $a$ of this source represents in some phenomenological way the radius of the coherent structures before their collapse, and will be assumed to be of the same order as the radius $a^{\prime}$ of the body. The amplitude $h$ of the oscillations is just a device to reproduce experimentally a source of oscillatory strength and, as this strength, 
depends on the precise dynamics of the collapse. Hereafter these approximations will be used and primes will be omitted.

The main conclusion of Sections 2 and 3 was the strong dependence of the wave field on the frequency ratio $\Upsilon=\omega_{0} / N$, which can be expressed in terms of the Strouhal number $S t=a \omega_{0} / \pi U$ as $\Upsilon=\pi S t F r$. In the Reynolds and Froude numbers range considered in the literature, the Strouhal number is 0.2 for towed bodies and 1.0 for self-propelled bodies (see, e.g., Voisin, 1994b). A sphere towed at a Froude number $F r=5$ corresponds thus to $\Upsilon=\pi$, value that we adopted in Figs. 3(b) and 4 to compute the axial wavelength and vertical displacement field, respectively; these are to be compared with Figs. 8 and 9 of Bonneton et al. (1993).

Comparison between the theoretical and experimental wavefronts has already been successfully performed by Gilreath and Brandt (1985) and Bonneton et al. (1993) and will not be repeated here. As this wavefront is passed all waves are observed nearly simultaneously, with axial wavelengths close to one another and tending rapidly towards the asymptotic values (10), which themselves do not differ much from the spatial period $2 \pi U / \omega_{0}$ of the wake. Then, consistently with Hopfinger et al. (1991), Bonneton et al. (1993) and Lin et al. (1993), a régime is reached in which the wavelength is independent of the Froude number and identical to the mean spacing of the coherent structures, which in terms of the diameter $2 a$ of the body means $\lambda_{x} / 2 a \sim S t^{-1} \approx 5$

It is tempting to associate this régime with the coherent wave régime reported by Bonneton et al. (1993) and already distinguishable in the results of Hopfinger et al. (1991). In particular, $\lambda_{x} / 2 a$ is roughly of the same order as the wavelength of the first coherent waves identified by Bonneton et al. (1993). However, the associated wave field, shown in Fig. 4, exhibits a very complicated phase structure resulting from the interference between the four wave systems, 
and differs from the more or less organized structure observed by Hopfinger et al. (1991) and Bonneton et al. (1993). This makes the present interpretation just tentative.

We finally point out that the analysis exposed in the present paper relies entirely on the assumption that advection of the coherent structures before the collapse is negligible, so that the process of emission of the waves is impulsive. There may, however, be situations where advection is significant before the collapse. Then each structure generates not an impulsive wave field but a lee wave field, as reported by Sysoeva and Chashechkin (1991) for a towed sphere and by Dupont and Kadri (1994) for a bell-shaped obstacle.

\section{ACKNOWLEDGEMENTS}

The authors gratefully acknowledge the advice and guidance of Prof. E.J. Hopfinger. Experiments were carried out by one of the authors (P. D.) during a one-year stay within the SPEA team of the Centre National de Recherches Météorologiques; thanks to all of them for their kindness and invaluable help. 


\section{REFERENCES}

Abramowitz, M. and Stegun, I.A. (Editors), 1972. Handbook of Mathematical Functions. Dover, New York, 1060 pp.

Bonneton, P., Chomaz, J.-M. and Hopfinger, E.J., 1993. Internal waves produced by the turbulent wake of a sphere moving horizontally in a stratified fluid. J. Fluid Mech., 254: 23-40.

Davies, P.A., Boyer, D.L., Fernando, H.J.S. and Zhang, X., 1994. On the periodic motion of a circular cylinder through a linearly stratified fluid. Phil. Trans. R. Soc. Lond., Ser. A, 345: $353-386$.

Dupont, P. and Kadri, Y., 1994. Internal waves generated by the wake of a Gaussian mountain. (In preparation).

Gilreath, H.E. and Brandt, A., 1985. Experiments on the generation of internal waves in a stratified fluid. AIAA J., 23: 693-700.

Gorodtsov, V.A. and Teodorovich, E.V., 1982. Study of internal waves in the case of rapid horizontal motion of cylinders and spheres. Fluid Dyn., 17: 893-898.

Graham, E.W. and Graham, B.B., 1980. The tank wall effect on internal waves due to a transient vertical force moving at fixed depth in a density-stratified fluid. J. Fluid Mech., 97: 91-114.

Hopfinger, E.J., Flor, J.-B., Chomaz, J.-M. and Bonneton, P., 1991. Internal waves generated by a moving sphere and its wake in a stratified fluid. Exp. Fluids, 11: 255-261.

Lighthill, J., 1978. Waves in Fluids. Cambridge University Press, Cambridge, 520 pp. 
Lin, J.-T. and Pao, Y.-H., 1979. Wakes in stratified fluids. Ann. Rev. Fluid Mech., 11: 317-338.

Lin, Q., Boyer, D.L. and Fernando, H.J.S., 1993. Internal waves generated by the turbulent wake of a sphere. Exp. Fluids, 15: 147-154.

Miles, J.W., 1971. Internal waves generated by a horizontally moving source. Geophys. Fluid Dyn., 2: 63-87.

Peat, K.S. and Stevenson, T.N., 1975. Internal waves around a body moving in a compressible density-stratified fluid. J. Fluid Mech., 70: 673-688.

Redekopp, L.G., 1975. Wave patterns generated by disturbances travelling horizontally in rotating stratified fluids. Geophys. Fluid Dyn., 6: 289-313.

Rehm, R.G. and Radt, H.S., 1975. Internal waves generated by a translating oscillating body. J. Fluid Mech., 68: 235-258.

Stevenson, T.N. and Thomas, N.H., 1969. Two-dimensional internal waves generated by a travelling oscillating cylinder. J. Fluid Mech., 36: 505-511.

Sysoeva, E.Ya. and Chashechkin, Yu.D., 1991. Vortex systems in the stratified wake of a sphere. Fluid Dyn., 26: 544-551.

Voisin, B., 1994a. Internal wave generation in uniformly stratified fluids. Part 2. Moving point sources. J. Fluid Mech., 261: 333-374.

Voisin, B., 1994b. Internal wave generation by turbulent wakes. In: J.M. Redondo and O. Métais (Editors), Mixing in Geophysical Flows. Universitat Politècnica de Catalunya, Barcelona, pp. 291-301. 


\section{FIGURE CAPTIONS}

Fig. 1. Determination of the wave field of a horizontally translating source of oscillatory strength. (a) System of coordinates; (b) graphical solution of equation (8) for the parameter $\xi_{*}=\xi /\left|\sin \varphi_{1}\right|$, for $\Upsilon /\left|\sin \varphi_{1}\right|=0.5(-)$ and $\Upsilon /\left|\sin \varphi_{1}\right|=1.2(---)$.

Fig. 2. Vertical displacement field generated by horizontal translation of a vertically oscillating sphere, for $\Upsilon=0.4,0.8$ and 1.2, with $F r=0.25$ and $K e=0.6$. Dimensionless coordinates $\mathbf{r}_{*}=$ $N \mathbf{r}_{1} / U$ are used, with $z_{*}=24$ (except for $\Upsilon=0.4$, in which case $z_{*}=17$ ). (a) shows the first few curves of constant phase of each wave system (__ $)$ and the associated wavefronts $\left({ }_{-}-{ }_{-}\right)$; for $\Upsilon<1$ these fronts comprise a circle, whose upstream and downstream halves correspond to divergent and transverse sum waves, respectively. The theoretical displacement field is shown in (b) for the dipole and in (c) for the surface source, while (d) is the experimental visualization; gray scale units are arbitrary.

Fig. 3. Dimensionless axial wavelength $\lambda_{*}=\left(N \lambda_{x}\right) /(2 \pi U)$ versus dimensionless axial distance $x_{*}$ in the plane $y_{*}=0$, for $\Upsilon=0.6$ and $z_{*}=24$ (a) and $\Upsilon=\pi$ and $z_{*}=0.6$ (b). _ the source path.

Fig. 4. Vertical displacement field generated by horizontal translation of a vertically oscillating sphere, for $\Upsilon=\pi, F r=5$ and $z_{*}=0.6$. 


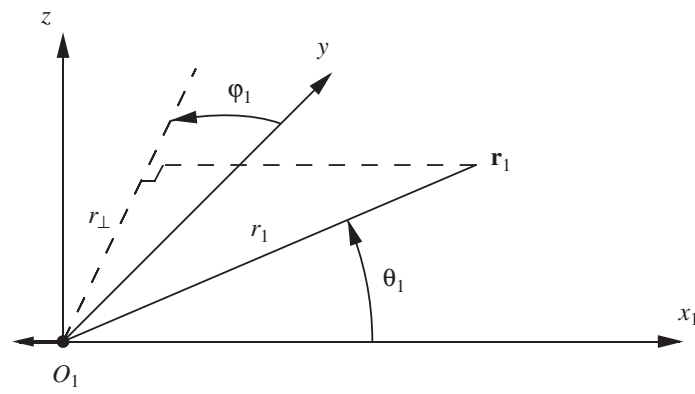

(a)

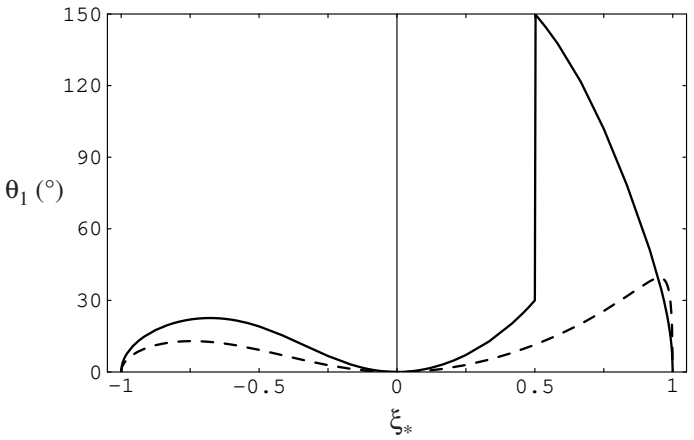

(b)

Fig. 1. Determination of the wave field of a horizontally translating source of oscillatory strength. (a) System of coordinates; (b) graphical solution of equation (8) for the parameter $\xi_{*}=\xi /\left|\sin \varphi_{1}\right|$, for $\Upsilon /\left|\sin \varphi_{1}\right|=0.5(-)$ and $\Upsilon /\left|\sin \varphi_{1}\right|=1.2(---)$. 
(a)
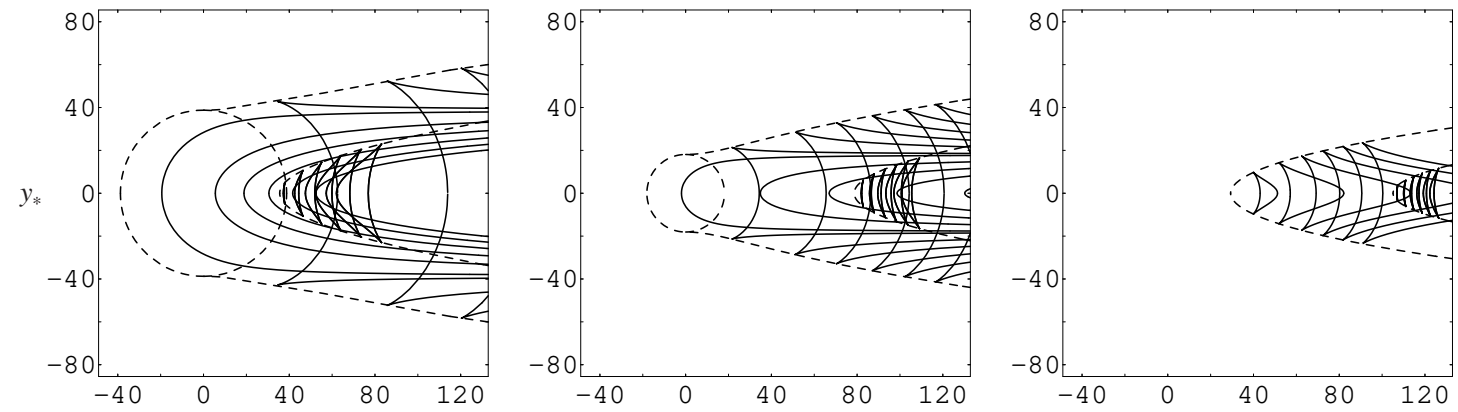

(b)
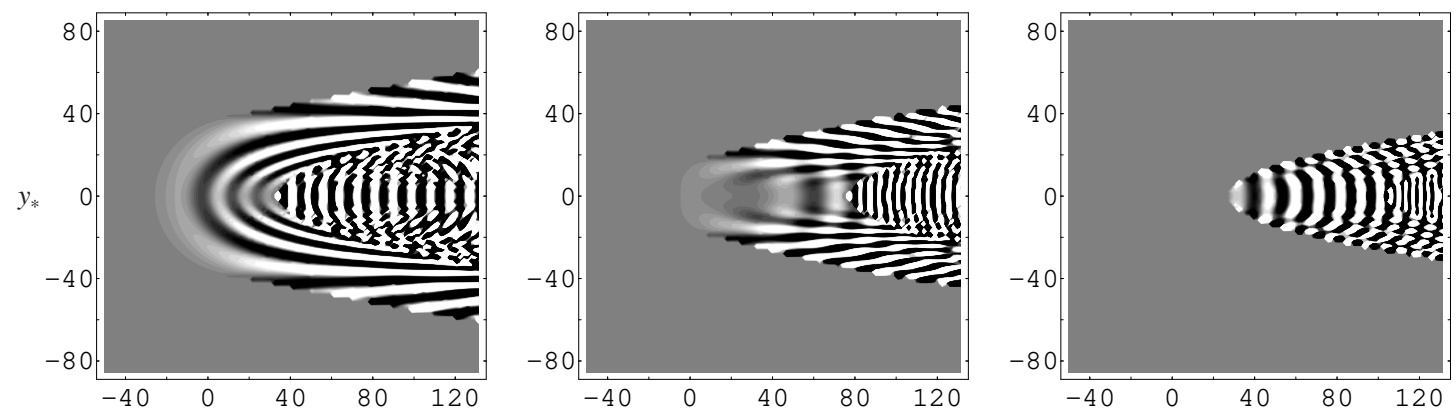

(c)
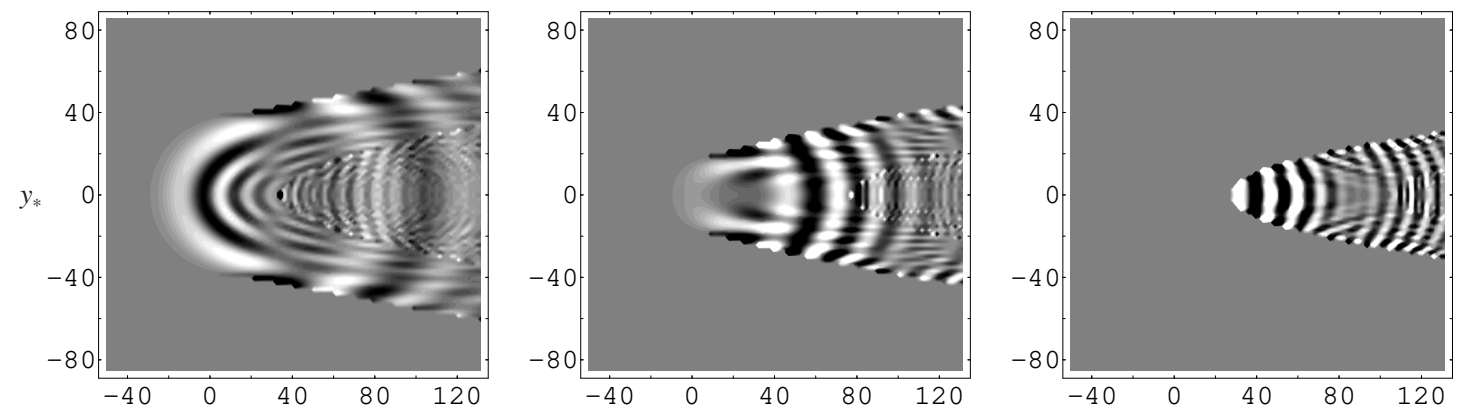

(d)
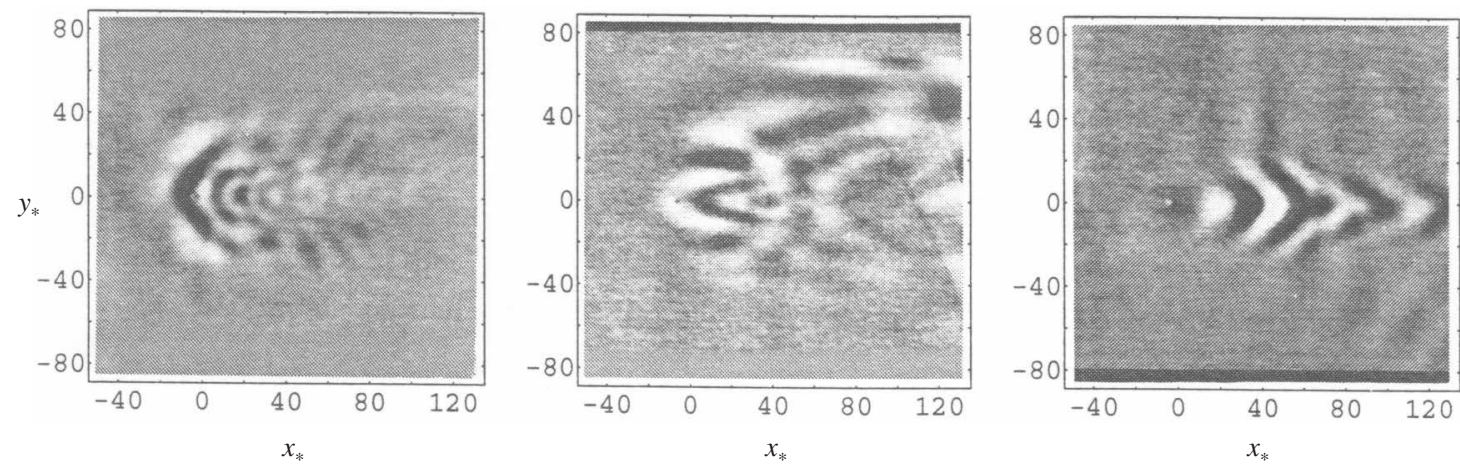

$\Upsilon=0.4$

$\Upsilon=0.8$

$\Upsilon=1.2$

Fig. 2. Vertical displacement field generated by horizontal translation of a vertically oscillating sphere, for $\Upsilon=0.4,0.8$ and 1.2, with $F r=0.25$ and $K e=0.6$. Dimensionless coordinates $\mathbf{r}_{*}=N \mathbf{r}_{1} / U$ are used, with $z_{*}=24$ (except for $\Upsilon=0.4$, in which case $z_{*}=17$ ). (a) shows the first few curves of constant phase of each wave system (- ${ }_{-}$) and the associated wavefronts $(---)$; for $\Upsilon<1$ these fronts comprise a circle, whose upstream and downstream halves correspond to divergent and transverse sum waves, respectively. The theoretical displacement field is shown in (b) for the dipole and in (c) for the surface source, while (d) is the experimental visualization; gray scale units are arbitrary. 


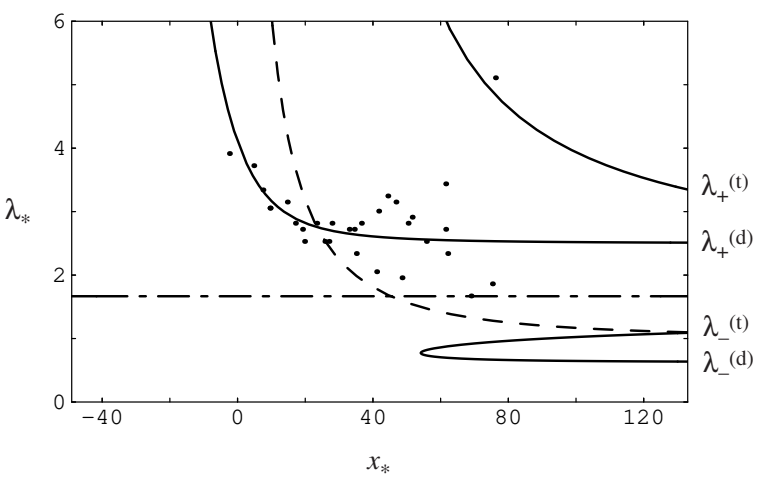

(a)

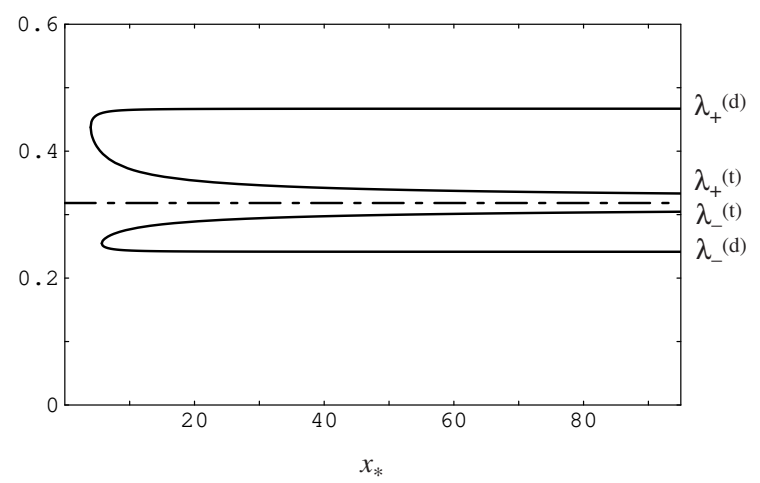

(b)

Fig. 3. Dimensionless axial wavelength $\lambda_{*}=\left(N \lambda_{x}\right) /(2 \pi U)$ versus dimensionless axial distance $x_{*}$ in the plane $y_{*}=0$, for $\Upsilon=0.6$ and $z_{*}=24$ (a) and $\Upsilon=\pi$ and $z_{*}=0.6$ (b). — Theoretical curves; $\cdot$, experimental points; - - -, lee wave length; _- _, spatial period of the source path.

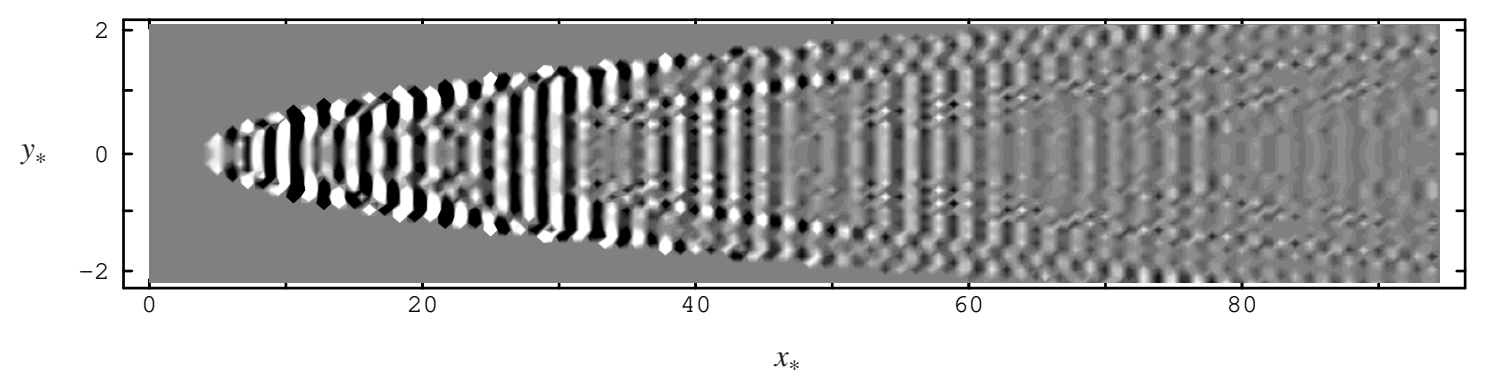

Fig. 4. Vertical displacement field generated by horizontal translation of a vertically oscillating sphere, for $\Upsilon=\pi, F r=5$ and $z_{*}=0.6$. 